\title{
PERSPECTIVE
}

\section{The 'omics revolution and our understanding of sperm cell biology}

\author{
Mark A Baker
}

The foundations of proteomics are to study gene products and their regulatory roles within cells. Paradoxically, the only evidence that sperm cells make new proteins is through mitochondrial protein synthesis. Yet despite this, spermatozoa are the perfect candidates for mass spectrometry and hence, proteomic analysis. These enterprising cells use a plethora of post-translational modifications in order to gain functionality following their production within the testis. By using a combination of two-dimensional polyacrylamide gel electrophoresis (2D-PAGE), and more recently liquid chromatography-mass spectrometry (LC-MS)/MS, recent advances in sperm cell biology, through the use of proteomics, is making unparalleled progress. The protein inventory lists being generated have shed light on transmembrane proteins, kinases and chaperones never previously recognized. In addition, the ability to isolate either phosphopeptides or glycopeptides and quantify the differences between cells of two different populations make proteomic analysis of spermatozoa a real chance to finally answer some age old questions.

Asian Journal of Andrology (2011) 13, 6-10; doi:10.1038/aja.2010.62; published online 25 October 2010

Keywords: capacitation; DIGE; epididymis; LC-MS; proteomics; quantitation; sperm maturation; spermatozoa

\section{SPERMATOZOA, A 'SILENT' ACHIEVER}

Truth can often be found only after studying both extremes of an argument. The use of different 'omics approaches to study sperm cell biology, whether it be gene- or protein-based, will depend on the fundamental belief of when protein translation is, or is not, turned off. The thesis often put forward, is that sperm cells are transcriptionally silent. The antithesis held by others is that sperm cells are not only capable, but do require transcription for successful fertilization. ${ }^{1}$ In support of the former, it is recognized that following spermatogenesis, there is a twofold reduction in ploidy, which precedes chromatin remodeling though the sequential replacement of histones with transition proteins, and transition proteins with protamines. ${ }^{2-5}$ The latter packages DNA so tightly that it is unlikely to allow binding of transcription factors, leading to silencing of the DNA. ${ }^{6}$ Indeed experimentally, incubation of rabbit spermatozoa with radiolabeled leucine demonstrated no uptake, leading the authors to conclude that no protein synthesis was taking place within spermatozoa of this species. $^{7}$ However, in what seems to be a contradiction, by adding a mixture of $\left[{ }^{3} \mathrm{H}\right]$-amino acids to washed $\mathrm{Balb} / \mathrm{c}$ mouse spermatozoa, incorporation of activity appeared to increase linearly over a 3-h time period. ${ }^{8}$ This activity was shown to be sensitive to the mitochondrial ribosomal protein synthesis inhibitor chloramphenicol, but not the cytoplasmic protein synthesis inhibitor, cyclohexamine, suggesting mitochondrial involvement. ${ }^{8}$ Supporting this argument, autoradiographs of mouse sperm following incubation clearly showed that the radiolabeled amino acids localized to the sperm midpiece. Furthermore, SDS-polyacrylamide gel electrophoresis (PAGE) demonstrated that $\left[{ }^{3} \mathrm{H}\right]$-amino acids were incorporated into full length proteins, and therefore, the activity was not a result of free amino acids storage with the sperm cells. The observation that spermatozoa produce new proteins using mitochondrial ribosomes has also been shown in other species. For example, Premkumar and Bharagava reported protein synthesis by bovine sperm, which again was inhibited by chloramphenicol and not cycloheximide. ${ }^{9}$ Furthermore, Mujica ${ }^{10}$ have shown evidence for human sperm protein biosynthesis and finally Gur and Breitbart have recently demonstrated incorporation of $\left[{ }^{35} \mathrm{~S}\right]$-methionine and $\left[{ }^{35} \mathrm{~S}\right]$-cysteine into newly synthesized proteins of sperm during 'capacitation' of either human, mouse, bovine or rat spermatozoa. ${ }^{1}$ In this latter report, incorporation of radioactivity was suppressed by chloramphenicol, tetracycline and gentamycin (all mitochondrial ribosomal inhibitors), but not cycloheximide. So in summary, a number of independent laboratories have clearly demonstrated mitochondrial production of proteins with spermatozoa of several species. The canonical view of the latter, is that mitochondrial DNA encodes for 13 proteins (seven from complex 1, one from complex 3 , three from complex 4 and two from complex 5), all of which are known. ${ }^{11}$ The controversy at this present time, is not whether sperm mitochondrion produce new proteins (and theoretically, given that the mitochondrial DNA is not protaminated, there appears to be little reason why either transcription or translation within the mitochondria does not continue as per a somatic cell), but which proteins are being produced. Gur and Breitbart have argued that the addition of chloramphenicol stops the production of 22 proteins, ${ }^{1}$ many of which have been shown by others to have a critical involvement in sperm capacitation, including the sperm-egg fusion receptor Izumo. What's novel and controversial about these 
22 proteins, is that none are the 13 canonical ones derived from mitochondrial ribosomes. Making this concept even more challenging, it is difficult to conceptualize how some of the 'new' proteins being produced are handled by the cell. For example, how does the structural protein, A-kinase-anchoring protein 4 (AKAP4), which is normally insoluble, be transported through the mitochondria to the sperm flagella? Furthermore, if AKAP4 is produced, then why do others not see the radiolabeled signal within the sperm flagellum? Unfortunately, the authors failed to produce the raw data necessary to judge their interpretation. For these reasons, and the ability of pharmacology intervention, including kinase inhibitors to block sperm function, ${ }^{12,13}$ it is still the view of many today, that further maturation of the sperm cell, following elongation, is dominated through post-translational modifications. It is to this end, that proteomics, and not transcriptomics, stands alone as the dominant force to study sperm maturation.

\section{THE MOST PROFOUND STATEMENTS ARE OFTEN SPOKEN IN SILENCE}

Although the testicular environment develops a morphological sperm cell, paradoxically, this cell is incapable of movement, recognizing the egg and undergoing the acrosome reaction. Terminally differentiated and in an autonomous state, the male gamete can no longer rely on further protein biosynthesis as a means for maturation. Thus, the pivotal question raised to reproductive biologist is how to study a cell that essentially does not speak.

The different fields of 'omics have indeed tried to answer this question. Remarkably, several laboratories have investigated the human sperm transcriptome, through both microarray-based experiments and serial analysis of gene expression analysis. Both have concluded that the mRNA species present within sperm must be either from past events; the gene expression of spermatozogenesis, or for providing the zygote with unique suite of paternal mRNAs. ${ }^{14-17}$

In contrast to transcriptomics, the use of proteomics in sperm biology plays a unique, and arguably, pivotal role unseen in any other tissue/cell type beside blood. However, unlike blood, two important parameters make sperm cell an outstanding candidate for proteomic analysis. The dynamic range (concentration difference between the most abundant protein and the least), is around $10^{12} \log$ orders of magnitude in blood (albumin versus interferon). ${ }^{18}$ In contrast, the most abundant proteins in spermatozoa (AKAP4), is only expressed around $10^{5-6}$ orders of magnitude, compared to the (known) lowest proteins (phospholipase- $\zeta$ ). Furthermore, the number of proteins expressed in blood is estimated to be around 6000. This is much higher compared to the best estimates for spermatozoa, which are between 2500 and 3000 proteins. ${ }^{19}$ In addition to these factors, sperm cells can be obtained in extremely high purity and at large concentrations, making them arguably the best cell type most adapted to proteomic analysis.

Yet despite this compendium of arguments, one fact beyond all else will necessitate that proteomic analysis of spermatozoa will continue for many years to come. This is that these cells rely on posttranslational modifications as a major driving force for post-testicular maturation. ${ }^{19}$ At present, the most suitable means of characterizing these events is through mass spectrometry (MS).

\section{THE SECRETS OF A SILENT CELL}

Initial attempts to identify those proteins present within sperm began at the two-dimensional (2D)-PAGE level. ${ }^{20}$ The dynamic range of this technology lies between $10^{3}$ and $10^{4}$ orders of magnitude, depending on whether fluorescent dyes are used for protein detection. In practice, this means that even when using narrow $\mathrm{pH}$ ranges, only a small percentage of the proteome is visualized. ${ }^{21}$ Nonetheless, the earliest attempts at defining the human proteome found 1000 protein spots, of which, 98 unique proteins were identified by matrix-assisted laser desorption/ionization-time of flight (MALDI-ToF-ToF)-MS. ${ }^{22,23}$ Greater coverage of the sperm proteome was demonstrated in the human, when newer technologies, including 2D-liquid chromatography (LC), directly coupled to an ion-trap mass spectrometer were employed. ${ }^{24}$ Here, pooled human sperm ejaculates were purified using Percoll gradients, then subject to $1 \%$ Triton X-100 solubilization. ${ }^{24}$ This has the advantage that the soluble proteins would not be saturated with the higher abundant proteins including AKAP4, tubulin and outer dense fibers, which typically require a harsher lyses approach. Thus, the sample complexity and dynamic range is reduced. Furthermore, the insoluble pellet was subject to SDS-PAGE extraction, then both the Triton-X 100 soluble and insoluble proteins were run on an SDS gel. Gel strips were taken every $2 \mathrm{~mm}$. Due to the limitations in mass scanning range (typically around 50-6000 Da), peptides are often used for what is known as 'bottom-up' sequencing approach. For this cause, trypsin digestion of the gel strips is performed, and the peptides are then injected onto a reversed phase column and eluted directly into the MS. The latest state-of-the-art mass spectrometers can handle up to $10^{5}$ orders of magnitude, again, well below $10^{9}$ needed for a whole-system approach. Nevertheless, in the human, 1056 proteins were positively identified. The identification of these proteins presented some interesting facts, which either reenforced current concepts or introduced novel ideas into reproductive biology not previously considered. For example, 437 proteins were shown to belong to various metabolic pathways within spermatozoa. Not only does this demonstrate a huge reliance on glycolysis, but also these data suggest that glycolytic proteins in particular are amongst the highest expressed proteins with human spermatozoa. Before the sequencing of the human sperm proteome was undertaken, very little was known about the surface receptors with sperm cells. However, at least six 7-transmembrane protein receptors and six tyrosine kinase receptors were detected. One receptor that is of prolactin has recently been studied in further detail. ${ }^{25}$ This protein was found to be localized to the postacrosomal region of the sperm head, neck midpiece and principal piece of the tail. Interestingly, addition of prolactin was shown to be a prosurvival factor for human spermatozoa. Hence, in the presence of $25-1000 \mathrm{ng} \mathrm{ml}^{-1}$ prolactin, up to $50 \%$ of human sperm cells continued to be motile in a basic salt solution, compared to only $5 \%$ of the vehicle control after $19 \mathrm{~h} .^{25}$ Interestingly, direct measurement of the level of prolactin in epididymal plasma recorded a level of $650 \mathrm{ng} \mathrm{ml}^{-1}$, 25 suggesting that prolactin plays a survival role within the epididymal lumen.

The amount of proteins identified involved in post-translational modification or protein (re)folding events was astonishing. Included in this inventory were heat shock proteins (HSPs) 10, 40, 70, 72, 75 and $90{ }^{26}$ Furthermore, HSP60 has been detected in other species. ${ }^{19,26,27}$ Why do sperm carry around so many HSPs within their proteome? And, as is the case for HSP70 and HSP90, why are they expressed at such high levels? The answers may be as intriguing as the question. Several HSPs, including HSP60, HSP90 and HSP70, undergo phosphorylation during capacitation. ${ }^{28-34}$ Recent evidence has shown that HSP60 and HSP90 (ERp99 and Grp94) migrate to the surface of the sperm head during capacitation, in concert with the ability of sperm to bind to the egg. In addition, although circumstantial, it was interesting to note that the only tyrosine-phosphorylated sperm heads were able to bind to the zona pellucida. Since the addition of anti-HSP60 or 
-HSP90 antibodies did not inhibit sperm-zona pellucida binding, the authors have speculated that HSPs on the sperm surface are involved in formation of a complex, which is ultimately responsible for zona pellucida interaction. ${ }^{35}$

Apart from the human, other large scale studies utilizing LC-MS have been performed on rat $^{27}$ mouse, ${ }^{26}$ bull $^{36}$ and fruit fly ${ }^{37}$ sperm cells. An analysis of the 'common' proteins present within these species (common being found in at least two species) has been performed. ${ }^{19}$ From these lists, several classes of proteins/enzymes were found. For example, no less than 18 different kinases were found to be common between the human, rat and mouse sperm proteomes. One remarkable facet was the different isoforms of protein kinase A (PKA) present within sperm cells. This enzyme has been reported to play a role in just about every aspect of sperm function that has been studied. ${ }^{12,13,38-42}$. PKA is a tetramer, consisting of both regulatory and catalytic subunits. By using a molecular approach, deletion of the regulatory type II $\alpha$ regulatory subunit delocalized PKA in the mouse sperm, but did not affect motility or fertility. ${ }^{43}$ Interestingly, sperm proteomic analyses have suggested that two other types of PKAregulatory subunits are present, including $\mathrm{RI} \alpha$ and RII $\alpha$. Haploinsufficiency of the latter gives rise to fertility defects in male mice and men. ${ }^{44}$ Thus, it has been suggested that there is some redundancy toward the catalytic subunit. ${ }^{40}$ Genetic approaches investigating the PKA-catalytic subunits have demonstrated the importance of this enzyme. By removing the PKAc $\alpha$ gene (and abolishing both PKA $\alpha 1$ - and PKA $\alpha$ II-catalytic subunits), a small percentage of knockout mice survived to adulthood. ${ }^{38}$ These spermatozoa had normal spermatogenesis, but defects in forward motility, with a neartotal loss in progressive movement. By measuring enzyme activity, the authors could demonstrate that the PKAc $\alpha$ knockout mice essentially had little to no PKA activity in sperm, suggesting that PKAc $\alpha$ was indeed the major kinase(s) present within these cells. To sharpen this approach, individual catalytic subunits of PKA have since been targeted. Deletion of the PKC $\alpha$ II produced mice whose spermatzoa swim spontaneously in vitro, suggesting that this particular catalytic subunit has no role in the formation of a functional flagellum of the initiation of motility. However, the protein was necessary to speed the flagella beat, and for the tyrosine phosphorylation of proteins normally seen during capacitation. ${ }^{45}$ To my knowledge, no report exists on sperm PKA $\alpha$ I knockout mice; however, a chemical-genetic approach has been applied to sperm PKA $\alpha$. Herein, the ATP-binding socket of the gene products ( $\alpha \mathrm{I}$ and $\alpha \mathrm{II}$ ) are made susceptible to the chemical 4-amino-5-(4-methylphenyl)-7-( $t$-butyl)pyrazolo[3,4- $d$ ]-pyrimidine (PP1) through an M120A substitution. Addition of PP1 did not stop forward progressive motility per se; however, it did stop an increase in forward progressive motility usually seen upon addition of $\mathrm{HCO}_{3}^{-}$ (see Figure 3 of Ref. 46). This raises the question of whether either isoform of PKA $\alpha$ is responsible for the initiation of motility as suggested by the PKA $\alpha$ knockout or could there be evidence for the involvement of another PKA isoform. The answer may lie in the proteomic analysis of the rat sperm cells. ${ }^{27}$ Herein, a non-redundant peptide hit (i.e., a peptide that only matches to one isoform), was found for the catalytic subunit of PKA $\beta$. Hence, it is tempting to speculate that while the PKA $\alpha$ global knockout produced sperm that were essentially immotile, this is more likely a developmental defect, rather than the involvement of any of the PKA $\alpha$ isoforms in motility activation per se. Hence, PKA $\beta$ may be responsible for the initial onset of motility, whilst PKA $\alpha 1$ then gives a 'boost' to the forward progressive motility. ${ }^{46}$ Finally, PKA $\alpha$ II appears to be responsible for the hyperactivated movement necessary for sperm fertilization.

\section{MAKING SPERMATOZOA SING}

The creation of proteomic lists within spermatozoa gives us a 'snapshot' or inventory of proteins present within the cell. However, functionally, it is still difficult to ascertain which of these proteins are, for example, modified during epididymal transit to allow sperm-egg binding, or initiate sperm motility. To answer these questions, comparative proteomic analysis has to be performed. In difference to simply identifying a protein, quantitative proteomic analysis requires a higher degree of expertise.

Initial comparisons of sperm proteomes were again performed at the level of 2D-PAGE, with 47 infertile patients with a cohort of fertile donors compared. From this study, 67 spots were found to undergo a change between the groups. ${ }^{47}$ However, the significant variation between the gels has led to criticism of the analysis. ${ }^{19}$ A second attempt at 2D-PAGE profiling comparing normal sperm with that of a patient who experienced in vitro fertilization failure found 20 differential spots, of which, actin-binding protein and outer dense fiber 2 could be identified. ${ }^{48}$ To overcome the inherent difficulties and reproducibility problems of 2D-PAGE, a less complex sample is desirable. Thus, 2D-PAGE applied to the nucleus of spermatozoa demonstrated 12 spots corresponding to protamines $\mathrm{P} 1$ and $\mathrm{P} 2 .{ }^{49}$ A quantitative analysis of these spots demonstrated a higher P1 to P2 level in two of the 184 men undergoing male infertility diagnosis. ${ }^{50,51}$

A second way to overcome the inherent difficulties of 2D-PAGE is to use internally labeled fluorescent dyes, in the process now known as 'difference in gel electrophoresis' or DIGE. This technology allows three samples to be run simultaneously within a $2 \mathrm{D}$ gel. One sample is comprised of a pooled internal standard, and if run correctly, generates a standard for every protein spot detected, from which to accurately quantitate protein levels. By comparing rat spermatozoa from the cauda and caput epididymis, 60 protein spots were shown to exhibit significant changes using the DIGE technology. ${ }^{52}$ Of these eight proteins were identified by MALDI-TOF-MS, including one protein undergoing serine phosphorylation as demonstrated by $2 \mathrm{D}$ western blotting.

The power of quantification is not limited to 2D-PAGE, but can also be applied to LC-MS technology, in perhaps a more versatile manner. Following tryptic digestion of proteins to produce peptides, several techniques are available to enrich for phosphopeptides, including the use of immoblized metal affinity chromatography. In a recent study, both non-capacitated and capacitated sperm digested were subject to phosphopeptide enrichment. Then, peptides were labeled using $\mathrm{d}_{0}$ and $\mathrm{d}_{3}$ methyl esters. This results in an increase in parent mass of the $d_{3^{-}}$ labeled peptide by $3 \mathrm{Da}$. Thus, for example, if a peptide had an original mass of $1000 \mathrm{Da}$, the $\mathrm{d}_{0}$ peptide would remain at this mass, whilst the $\mathrm{d}_{3}$-labeled peptide increases to $1003 \mathrm{Da}$. The samples were then combined and run through reversed phase, nanoflow chromatography, coupled to an ion-trap Fourier transform instrument. One major advantage of such labeling strategies is that the retention time of the labeled peptides under reversed phase conditions does not change. Hence, two co-eluting peptides will emerge from the c-18 column, with a difference of $3 \mathrm{Da}$. By integrating the peptide counts, it is very easy to perform a quantitative measurement and locate kinase-/phosphatase-regulated proteins. Using this approach, the laboratory of Visconti found 55 unique phosphorylation sites, of which 42 were said to change during capacitation. ${ }^{53}$ From this study, it is clear that phosphoregulation plays a major role in capacitation. For example, the regulatory binding domain within AKAP, underwent major phosphorylation changes, such that the R-Helix involved in binding PKArII demonstrated charge-density shifts. This is likely to affect the binding capacity of this regulatory domain to AKAP4. 
The use of such 'label' strategy for sperm proteomics is not limited to this technology. Indeed, other labels can be used, albeit they work in slightly different ways. For instance, ${ }^{18} \mathrm{H}_{2} \mathrm{O}$ can be used to label a sample, which results in an increased mass of the parent peptide by either 2 or $4 \mathrm{Da}$. Labeling occurs in the presence of trypsin. Again, the samples can then be mixed (multiplexed) and run through reversed phase, without affecting the retention times. Heavy water labeling quantitation is also based upon the integrated area of the parent mass peptide. Other labels amenable to sperm cell biology include isotopecoded affinity tag or isobaric tags for relative and absolute quantitation. The principal of increasing the mass of one peptide is the same; however, for both isotope-coded affinity tag and isobaric tags for relative and absolute quantitation, quantitation is performed at the MS/MS level and can only be used on high resolution, time of flight instruments including a Qq-ToF or MALDI-ToF-ToF.

The advantage of a global, quantitative proteomics approach is that the method is unbiased and capable of generating large amounts of meaningful data in a relatively short time frame. Coupled with techniques, including phosphopeptide enrichment through either immoblized metal affinity chromatography of titanium dioxide ${ }^{54,55}$ or indeed glycopeptides enrichment through the use of hydrazine, ${ }^{56}$ LC-MS-based proteomic analysis is ensured to be a technique that will be foundational in answering biological question related to sperm cells and their ability to undergo post-testicular maturation for successful fertilization.

\section{COMPETING FINANCIAL INTERESTS}

The authors declare no competing financial interests.

1 Gur Y, Breitbart H. Mammalian sperm translate nuclear-encoded proteins by mitochondrial-type ribosomes. Genes Dev 2006; 20: 411-6.

2 Zhao M, Shirley CR, Yu YE, Mohapatra B, Zhang Y et al. Targeted disruption of the transition protein 2 gene affects sperm chromatin structure and reduces fertility in mice. Mol Cell Biol 2001; 21: 7243-55.

3 Wouters-Tyrou D, Martinage A, Chevaillier P, Sautiere P. Nuclear basic proteins in spermiogenesis. Biochimie 1998; 80: 117-28.

4 Oliva R, Dixon GH. Vertebrate protamine genes and the histone-to-protamine replacement reaction. Prog Nucleic Acid Res Mol Biol 1991; 40: 25-94.

5 Balhorn R. The protamine family of sperm nuclear proteins. Genome Bio/2007; 8: 227.

6 Carrell DT, Emery BR, Hammoud S. Altered protamine expression and diminished spermatogenesis: what is the link? Hum Reprod Update 2007; 13: 313-27.

7 Hernandez-Perez O, Luna G, Reyes A. Re-evaluation of the role of spermatozoa as inducers of protein synthesis by the rabbit endometrium. Arch Androl 1983; 11: 239-43.

8 Bragg PW, Handel MA. Protein synthesis in mouse spermatozoa. Biol Reprod 1979 20: 333-7.

9 Premkumar E, Bhargava PM. Transcription and translation in bovine spermatozoa. Nat New Biol 1972; 240: 139-43.

10 Mujica A. Mitochondrial protein synthesis in human ejaculated spermatozoa. J Cell Biol 1976; 70: 416a.

11 Cummins J. Mitochondrial DNA in mammalian reproduction. Rev Reprod 1998; 3 $172-82$.

12 Baker MA, Aitken RJ. Identification of pp60c-src as a key PKA-stimulated tyrosine kinase involved in the capacitation and hyperactivation of murine spermatozoa. $J \mathrm{Ce} / \mathrm{l}$ Sci 2006; 119: 3182-92.

13 Baker MA, Hetherington L, Curry B, Aitken RJ. Phosphorylation and consequent stimulation of the tyrosine kinase $\mathrm{C}-\mathrm{Abl}$ by PKA in mouse spermatozoa; its implications during capacitation. Dev Biol 2009; 333: 57-66.

14 Nguyen MT, Delaney DP, Kolon TF. Gene expression alterations in cryptorchid males using spermatozoal microarray analysis. Fertil Steril 2009; 92: 182-7.

15 Ostermeier GC, Dix DJ, Miller D, Khatri P, Krawetz SA. Spermatozoal RNA profiles of normal fertile men. Lancet 2002; 360: 772-7.

16 Ostermeier GC, Goodrich RJ, Moldenhauer JS, Diamond MP, Krawetz SA. A suite of novel human spermatozoal RNAs. J Androl 2005; 26: 70-4.

17 Zhao Y, Li Q, Yao C, Wang Z, Zhou Y et al. Characterization and quantification of mRNA transcripts in ejaculated spermatozoa of fertile men by serial analysis of gene expression. Hum Reprod 2006; 21: 1583-90.

18 Simpson RJ, Bernhard OK, Greening DW, Moritz RL. Proteomics-driven cancer biomarker discovery: looking to the future. Curr Opin Chem Biol 2008; 12: 72-7.

19 Baker MA, Aitken RJ. Proteomic insights into spermatozoa: critiques, comments and concerns. Expert Rev Proteomics 2009; 6: 691-705.
20 Naaby-Hansen S, Flickinger CJ, Herr JC. Two-dimensional gel electrophoretic analysis of vectorially labeled surface proteins of human spermatozoa. Biol Reprod 1997; 56: 771-87.

21 Wulfkuhle JD, Liotta LA, Petricoin EF. Proteomic applications for the early detection of cancer. Nat Rev 2003; 3: 267-75.

22 Martinez-Heredia J, Estanyol JM, Ballesca JL, Oliva R. Proteomic identification of human sperm proteins. Proteomics 2006; 6: 4356-69.

23 Oliva R, de Mateo S, Estanyol JM. Sperm cell proteomics. Proteomics 2009; 9: 1004 17

24 Baker MA, Reeves G, Hetherington L, Muller J, Baur I et al. Identification of gene products present in Triton $\mathrm{X}-100$ soluble and insoluble fractions of human spermatozoa lysates using LC-MS/MS analysis. Proteomics Clin Appl 2007; 1 524-32.

25 Pujianto DA, Curry BJ, Aitken RJ. Prolactin exerts a prosurvival effect on human spermatozoa via mechanisms that involve the stimulation of Akt phosphorylation and suppression of caspase activation and capacitation. Endocrinology 2010; 151 1269-79.

26 Baker MA, Reeves G, Hetherington L, Aitken RJ. The mouse sperm proteome characterised via IPG strip prefractionation and LC-MS/MS identification. Proteomics 2008: 1720-30.

27 Baker MA, Hetherington L, Reeves G, Müller J, Aitken RJ. The rat sperm proteome characterised via IPG strip pre-fractionation and LC-MS/MS identification. Proteomics 2008; 8: 2312-21.

28 Boulanger J, Faulds D, Eddy EM, Lingwood CA. Members of the $70 \mathrm{kDa}$ heat shock protein family specifically recognize sulfoglycolipids: role in gamete recognition and mycoplasma-related infertility. J Cell Physiol 1995; 165: 7-17.

29 Dix DJ, Allen JW, Collins BW, Mori C, Nakamura N et al. Targeted gene disruption of Hsp70-2 results in failed meiosis, germ cell apoptosis, and male infertility. Proc Natl Acad Sci USA 1996; 93: 3264-8.

30 Ecroyd H, Jones RC, Aitken RJ. Tyrosine phosphorylation of HSP-90 during mammalian sperm capacitation. Biol Reprod 2003; 69: 1801-1807.

31 Erata GO, Kocak Toker N, Durlanik O, Kadioglu A, Aktan G et al. The role of heat shock protein 70 (Hsp 70) in male infertility: is it a line of defense against sperm DNA fragmentation? Fertil Steril 2008; 90: 322-7.

32 Kamaruddin M, Kroetsch T, Basrur PK, Hansen PJ, King WA. Immunolocalization of heat shock protein 70 in bovine spermatozoa. Andrologia 2004; 36: 327-34.

33 Volpe S, Galeati G, Bernardini C, Tamanini C, Mari G et al. Comparative immunolocalization of heat shock proteins (Hsp)-60, -70, -90 in boar, stallion, dog and cat spermatozoa. Reprod. Dom Anim 2008; 43: 385-92.

34 Zakeri ZF, Wolgemuth DJ. Developmental-stage-specific expression of the hsp70 gene family during differentiation of the mammalian male germ line. Mol Cell Biol 1987; 7 : 1791-6.

35 Nixon B, Bielanowicz A, McLaughlin EA, Tanphaichitr N, Ensslin MA et al. Composition and significance of detergent resistant membranes in mouse spermatozoa. J Cell Physiol 2009; 218: 122-34.

36 Peddinti D, Nanduri B, Kaya A, Feugang JM, Burgess SC et al. Comprehensive proteomic analysis of bovine spermatozoa of varying fertility rates and identification of biomarkers associated with fertility. BMC Syst Biol 2008; 2: 19.

37 Dorus S, Busby SA, Gerike U, Shabanowitz J, Hunt DF et al. Genomic and functional evolution of the Drosophila melanogaster sperm proteome. Nat Genet 2006; 38 1440-5.

38 Skalhegg BS, Huang Y, Su T, Idzerda RL, McKnight GS et al. Mutation of the Calpha subunit of PKA leads to growth retardation and sperm dysfunction. Mol Endocrinol 2002; 16: 630-9.

39 Schmitt JM, Stork PJ. PKA phosphorylation of Src mediates CAMP's inhibition of cell growth via Rap1. Mol Cell 2002; 9: 85-94.

40 Amieux PS, McKnight GS. The essential role of RI alpha in the maintenance of regulated PKA activity. Ann NY Acad Sci 2002; 968: 75-95.

41 Harrison RA. Rapid PKA-catalysed phosphorylation of boar sperm proteins induced by the capacitating agent bicarbonate. Mol Reprod Dev 2004; 67: 337-52.

42 Carr DW, Fujita A, Stentz CL, Liberty GA, Olson GE et al. Identification of spermspecific proteins that interact with A-kinase anchoring proteins in a manner similar to the type II regulatory subunit of PKA. J Biol Chem 2001; 276: 17332-8.

43 Burton KA, Treash-Osio B, Muller CH, Dunphy EL, McKnight GS. Deletion of type Ilalpha regulatory subunit delocalizes protein kinase $A$ in mouse sperm without affecting motility or fertilization. J Biol Chem 1999; 274: 24131-6.

44 Burton KA, McDermott DA, Wilkes D, Poulsen MN, Nolan MA et al. Haploinsufficiency at the protein kinase $A \mathrm{RI}$ alpha gene locus leads to fertility defects in male mice and men. Mol Endocrinol 2006; 20: 2504-13.

45 Nolan MA, Babcock DF, Wennemuth G, Brown W, Burton KA et al. Sperm-specific protein kinase A catalytic subunit Calpha2 orchestrates CAMP signaling for male fertility. Proc Natl Acad Sci USA 2004; 101: 13483-8.

46 Morgan DJ, Weisenhaus M, Shum S, Su T, Zheng R et al. Tissue-specific PKA inhibition using a chemical genetic approach and its application to studies on sperm capacitation. Proc Natl Acad Sci USA 2008; 105: 20740-5.

47 de Mateo S, Martinez-Heredia J, Estanyol JM, Dominguez-Fandos D, Vidal-Taboada $\mathrm{JM}$ et al. Marked correlations in protein expression identified by proteomic analysis of human spermatozoa. Proteomics 2007; 7: 4264-77.

48 Pixton KL, Deeks ED, Flesch FM, Moseley FL, Bjorndahl L et al. Sperm proteome mapping of a patient who experienced failed fertilization at IVF reveals altered expression of at least 20 proteins compared with fertile donors: case report. Hum Reprod 2004; 19: 1438-47. 
49 Yoshii T, Kuji N, Komatsu S, Iwahashi K, Tanaka Y et al. Fine resolution of human sperm nucleoproteins by two-dimensional electrophoresis. Mol Hum Reprod 2005; 11: 677-81.

50 de Yebra L, Ballesca JL, Vanrell JA, Corzett M, Balhorn R et al. Detection of P2 precursors in the sperm cells of infertile patients who have reduced protamine P2 levels. Fertil Steril 1998; 69: 755-9.

51 Oliva R. Protamines and male infertility. Hum Reprod Update 2006; 12: 417-35.

52 Baker MA, Witherdin R, Hetherington L, Cunningham-Smith $K$, Aitken RJ. Identification of post-translational modifications that occur during sperm maturation using difference in two-dimensional gel electrophoresis. Proteomics 2005; 5: 1003-12.
53 Platt MD, Salicioni AM, Hunt DF, Visconti PE. Use of differential isotopic labeling and mass spectrometry to analyze capacitation-associated changes in the phosphorylation status of mouse sperm proteins. J Proteome Res 2009; 8: 1431-40.

54 Thingholm TE, Jensen ON, Robinson PJ, Larsen MR. SIMAC (sequential elution from IMAC), a phosphoproteomics strategy for the rapid separation of monophosphorylated from multiply phosphorylated peptides. Mol Cell Proteomics 2008; 7: 661-71.

55 Thingholm TE, Larsen MR, Ingrell CR, Kassem M, Jensen ON. $\mathrm{TiO}_{2}$-based phosphoproteomic analysis of the plasma membrane and the effects of phosphatase inhibitor treatment. J Proteome Res 2008; 7: 3304-13.

56 Lewandrowski U, Sickmann A. N-glycosylation site analysis of human platelet proteins by hydrazide affinity capturing and LC-MS/MS. Methods Mol Biol 2009; 534: 225-38. 